\title{
Autism spectrum disorder traits among prisoners
}

\begin{tabular}{|r|l|}
\hline Journal: & Advances in Autism \\
\hline Manuscript ID & AIA-11-2015-0023.R1 \\
\hline Manuscript Type: & Research Paper \\
\hline Keywords: & Autism Spectrum Disorder, Prison, Forensic psychiatry, Assessment \\
\hline \multicolumn{2}{|l}{} \\
\end{tabular}

SCHOLARONE ${ }^{\text {m }}$

Manuscripts 


\section{Autism spectrum disorder traits among prisoners}

\section{Introduction}

It is thought that around $1 \%$ of the population have autism spectrum disorder (ASD). However, the male: female ratio of ASD of around 3:1 means that prevalence is higher among men $(2 \%)$ than women $(0.3 \%)$ and it has been estimated that up to $4.5 \%$ of single men have ASD (Brugha et al. 2009a). As such, we might expect higher rates of ASD and significant levels of ASD traits among populations of men who are likely to be single. Given the higher rate of ASD among individuals with an intellectual disability or borderline intellectual functioning we might also expect populations of men known to include a high proportion of those with lower IQ (for example, male prisoners) to have similarly higher rates of ASD and ASD traits.

There have been few studies specifically on ASD among prison populations (Underwood et al., 2013). Recent reviews highlight the need for further research to support the early identification of individuals with ASD within the prison setting (Allely, 2015; Robertson \& McGillivray, 2015; Lewis et al., 2015). Studies of offenders with ASD across the wider forensic and criminal justice services have produced mixed results when looking at prevalence (Sabet et al., 2015). In a Swedish study of young offenders referred for forensic assessment: $15 \%$ had ASD with further 12\% having probable ASD (Siponmaa et al., 2001), a US study in a maximum secure prison found that $4.4 \%$ of participants had significant ASD traits (Fazio et al., 2012) and Hare and colleagues (1999) found an ASD rate of $2.4 \%$ in three high secure hospitals in England while a study in the Scottish prison service found a rate of just $0.93 \%$ (Robinson et al 2012). Haw et al. (2013) looked at the characteristics of male patients in a low secure setting with ASD. Compared with those without ASD, they were more likely to be single, at a younger age at their first contact with psychiatric services and $73 \%$ had a psychiatric diagnosis (most commonly schizophrenia) but personality disorders and substance abuse were less common.Studies of ASD among forensic populations have produced mixed results (Sabet et al., 2015).

It is not proven that people with ASD have higher rates of offending (King \& Murphy, 2014), however, it is likely that those with co-occurring health and behaviour problems are more at risk of offending behaviour (Chaplin et al, 2013). There is evidence that challenging behaviour is highly prevalent among individuals with ASD (Holden et al. 2006). Aside from the repetitive and restricted behaviours that are characteristic of people with ASD, specific problems associated with ASD include aggression, self-injurious behaviour and hyperactivity (Levy et al. 2009; Tsiouris et al. 2003). These behaviours may lead individuals with ASD into contact with the CJS. Offenders with ASD have been associated with violent behaviour, sexual offending, fire setting, obsessive harassment (stalking) and computer/cyber-crimes (Ledingham \& Mills, 2015; Sabet et al., 2015). By contrast there is suggestion that individuals with ASD are less likely to break the law because they often have a preference rules which they stick to rigidly.

Individuals with ASD and other neurodevelopmental disorders are more vulnerable to bullying and may be less able to cope within forensic environments (McCarthy et al., 2015a; 
McCarthy et al., 2015b). Having ASD also has implications for release and parole; especially if there is an expectation of an individual to engage in treatment. Treatment programmes within prisons are often standardised; for many the problem is not an unwillingness to engage but not having the ability to take part in activities that require a higher level of ability.

Effective recognition and assessment of ASD is key to successful clinical intervention and service provision (National Audit Office 2009). It is common for individuals with significant ASD traits to remain unrecognised in adulthood; a barrier to assessment, diagnosis and intervention (Brugha et al. 2011; National Audit Office 2009). Therefore, it is important that the assessment of ASD takes place in adult populations, particularly those at high risk e.g. criminal justice and mental health services (Hayes 2002; Søndenaa et al. 2008). The aim of this study was to determine the extent of ASD traits among prisoners, the extent to which these had been previously recognised and the feasibility of screening for ASD among male prisoners.

\section{Methods}

This was a cross-sectional study set in a male prison (see McCarthy et al., 2015a; McCarthy et al., 2015b). The study took place in a London prison which was run by the National Offenders Management Service (NOMS), part of the Ministry of Justice, and had a maximum capacity of 798 prisoners.

\section{Participants}

Eligibility criteria for the study were that participants demonstrated capacity to give informed consent and had sufficient use of English to take part. The study was granted ethical approval by the NRES Committee North East - Northern \& Yorkshire (ref: 12/NE/0040) and NOMS approval by the Governor of HMP Brixton in March 2012.

Participants were recruited between May 2012 and June 2013 (full details of the recruitment process are described in McCarthy et al., 2015a \& 2015b). In total, 378 prisoners were approached to take part in the study and 240 consented to the screening interview. Among the 240 male prisoners who took part, $45 \%$ were from a Black or minority ethnic (BME) background. Participants were aged from 20 to 72 years with a mean of 34 .

\section{Measures}

The 20-item Autism Quotient (AQ-20) was used to measure traits of ASD among the participants (Brugha et al., 2011). Each item on this scale is rated zero or one with a score greater than or equal to 10 indicating the likely presence of ASD. The AQ-20 was developed for a national study on the prevalence of ASD in England (Brugha et al., 2009b). The AQ-20 is one tool among the many developed to screen for ASD (Sappok et al., 2015). It was chosen for its brevity and availability. Unlike the original studies that used the AQ-20 as a self-report tool, the current study used it in face-to-face interviews with participants and the items were rated by a researcher. 
Additional AQ-20 data from the 2007 Adult Psychiatric Morbidity Survey (APMS) was obtained from the UK Data Service (National Centre for Social Research, 2011). In this study, 7403 adults living in private households in England completed the AQ-20 (Brugha et al., 2009a).

Follow-up diagnostic assessment for ASD was carried out using the Autism Diagnostic Observation Schedule (ADOS; Lord et al., 1989) and, where possible, the Autism Diagnostic Interview (ADI-R; Lord et al., 1994). The ADOS and ADI-R are recommended as best practice for carrying out a diagnostic assessment for ASD (NICE, 2014). Adaptations were made to the standardised ADOS assessment so that it was acceptable for use in prison and to ensure the safety of the researchers (all interviews were carried out without prison staff present). As a consequence of establishment security rules, it was not possible to take the following ADOS items on to the prison wings and therefore these sections were omitted: construction task, book, pictures, cartoon pictures and creating a story objects. All other sections of the module four ADOS assessment were completed and participants were asked to create a story without using physical objects as props. The ADI-R was completed if a prisoner who screened positive for ASD gave consent for a parent to be contacted and a parent agreed to take part.

Participants were said to meet the study diagnostic criteria for ASD if they met the criteria for ASD using the ADOS and met the criteria for ASD using the ADI-R (where this was carried out). If neither of these assessments could be carried out, a participant was said to meet the study diagnostic criteria if there was evidence from their prison record that they had been clinically diagnosed with ASD.

The study also carried out follow-up diagnostic assessment on a sample of participants who scored $<10$ on the AQ-20. Of these participants were referred to the study by healthcare staff and were participants who reported they had previously been diagnosed or assessed for ASD. The remaining participants were selected using convenience sampling among prisoners who screened negative on the AQ-20 and were willing to take part in further assessment.

\section{Procedure}

The sample was recruited using two methods: approaching potential participants on the prison wings and by (staff or self) referral. For the latter sample, healthcare staff approached prisoners they thought may have ASD and asked if they were happy for their name to be passed on to the researchers so that they could explain the study to them. Information about the study and participant information sheets were disseminated throughout the prison by word of mouth and via peer support workers and a health and well-being day. Prisoners could approach researchers on the wing and ask to take part or they could indicate an interest in participating via staff. 
Where possible, the study was explained to prisoners in a private room where they were given a copy of the participant information sheet and consent form. If this was not possible, the study was explained to individuals on the prison Wing or in the presence of staff or other prisoners. Potential participants were always asked whether they were happy to discuss the study in this way. After being given time to read through the participant information themselves or with the help of the researcher, prisoners were asked whether would like time to think about participating and convey their decision to the researcher another day or if they were happy to make a decision about taking part right away. Potential participants were encouraged to discuss the study, participant information sheet and the consequences of taking part with others (e.g. health care staff, peer support workers, friends) if they wanted to. A structured tool developed by the researchers and used in previous studies was used to assess whether participants had capacity to consent (). Interviews were carried out in private rooms on the prison wings by trained researchers (no prison staff were present). Information was provided to those who met the study's criteria for ASD and these individuals were referred to prison mental health services for further clinical assessment.

Participants who screened positive for ASD were asked if they were willing to undergo further assessment. If there was time and the participant was happy to go ahead, this assessment took place immediately. More often, a convenient time was arranged to meet the participant again. Locating prisoners for follow up assessment could be difficult as they were not often in their cells. Access to the wings was restricted to two periods of around two hours per day. Prisoners would often be working on the wing or be off the wing in education or doing exercise. During association sessions it could be hard to find a participant as they could be anywhere on the wing/in someone else's cell. Further, participants were sometimes transferred to another prison before the researchers had been able to meet them again.

\section{Results}

ASD traits and diagnosis

The total AQ-20 scores of the 240 participants ranged from one to 15 with a mean total score of 6.74 (SD: 3). Figure 1 shows that the distribution of total AQ-20 scores was reasonably continuous (normal).

\section{Figure 1: distribution of total AQ-20 scores among the 240 participants.}

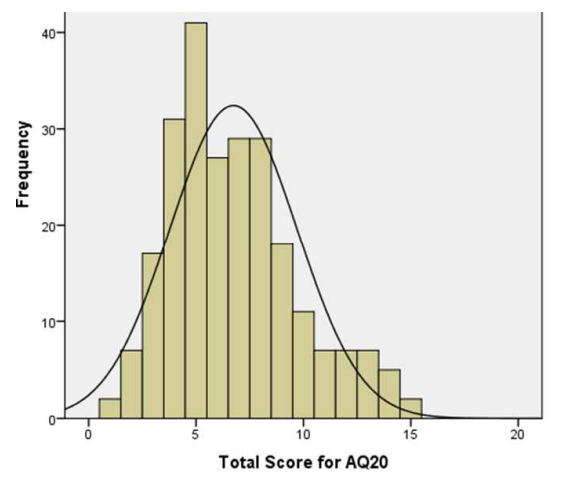


There were 39 participants with significant ASD traits according to the AQ-20 (total score $\geq 10$ ). Of the 39 participants who screened positive on the AQ-20, 32 underwent further assessment. Seven participants were lost to follow-up as they could not be contacted again for further diagnostic assessment. Of the 32 participants who were further assessed, 21 did not meet the study's diagnostic criteria for ASD. Among these participants were two who were assessed using the ADI-R: one met diagnostic criteria on the ADOS but not the ADI-R; and one could not be assessed using the ADOS but did not meet the diagnostic criteria using the ADI-R.

Eleven participants who screened positive for ASD met the study diagnostic criteria for ASD ( 8 met diagnostic criteria on the ADOS but an ADI-R could not be carried out and one met the diagnostic criteria on both the ADOS and ADI-R). Two participants were transferred out of the prison before further assessment could take place but were included in the ASD diagnosis group because they had an existing clinical diagnosis of ASD documented in their prison health record.

A further 22 participants who screened negative on the AQ-20 underwent further diagnostic assessment using the ADOS with three meeting the criteria for ASD. Nineteen did not meet diagnostic criteria for ASD. However, three did meet the criteria for ASD (using the ADOS). Two of these participants scored nine on the AQ-20 so narrowly missed screening positive. The other participant scored just three on the AQ-20 but had been referred by healthcare staff who thought he had some ASD traits. It was not clear why this participant's AQ-20 score was so low.

Thus, a large proportion (70\%), of those who screened positive for ASD on the AQ-20 did not meet diagnostic criteria and the AQ-20 resulted in three false negative assessments (a rate of $14 \%$ ). Sensitivity and specificity of the AQ-20 were $78.6 \%$ and $47.5 \%$, respectively. Internal consistency was poor (Cronbach's $\alpha=0.57$ ) and there were no significant correlations between the AQ-20 and the ADOS totals obtained from participants who were further assessed for ASD.

Rates of ASD among prisoners

Sub-analyses were carried out to explore the rate of ASD among the general prison population and among those who were identified by prison staff as having ASD difficulties. Figure 2 shows the distribution of AQ-20 scores among these two sampling groups. 
Figure 2: distribution of total AQ-20 scores among the 186 participants who approached on the prison wings and 54 who were staff or self-referred.

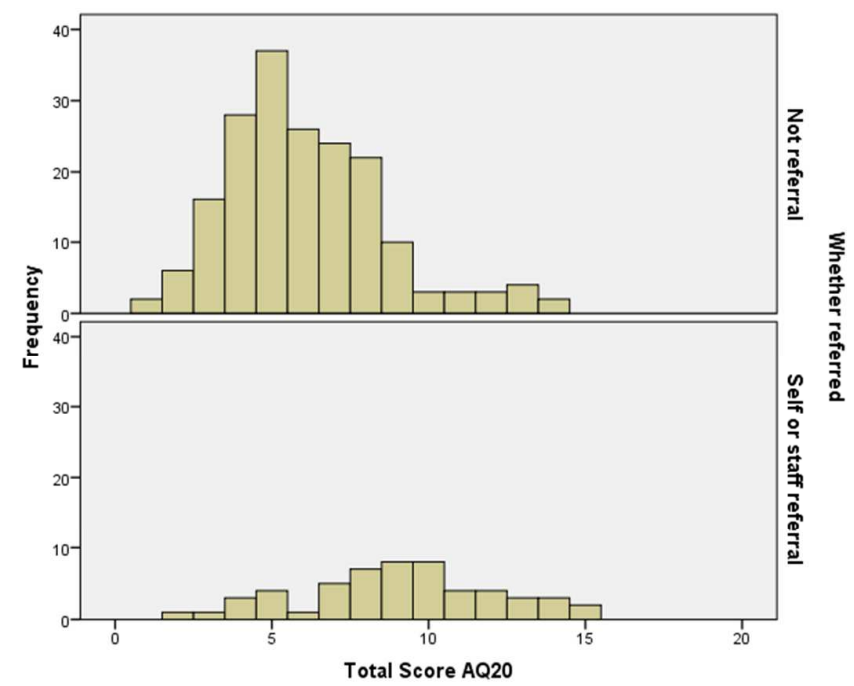

Rates of ASD among those who were approached on the prison wings ( $\mathrm{n}=186)$ were: $10 \%$ screened positive and $2 \%$ met diagnostic criteria. In the study prison, which has a capacity of 798 and where around 33 prisoners arrive each week, we estimate that one prisoner with significant ASD traits will arrive each week, 34 with ASD traits will arrive over one year and there will be around 16 prisoners with ASD traits in the prison at any one time.

The mean AQ-20 score for those approached on the prison wings was 6.06 (SD: 2.5). These results were compared with those for the 2618 men aged 20 to 72 years of age who completed the AQ-20 as part of the 2007 APMS (National Centre for Social Research, 2011). The mean total AQ-20 score for APMS participants was 6.25 (SD: 2.7). This is slightly higher than the mean total score of our prison sample but not statistically significantly (mean difference: 0.18 , 95\% CI: -0.21 to $0.58, p=0.36$ ). As with the current study AQ-20 scores were normally distributed (see figure 3 ).

Figure 3: distribution of total AQ-20 scores among the 2618 men aged 20 to 72 years of age who completed the AQ-20 as part of the 2007 APMS.

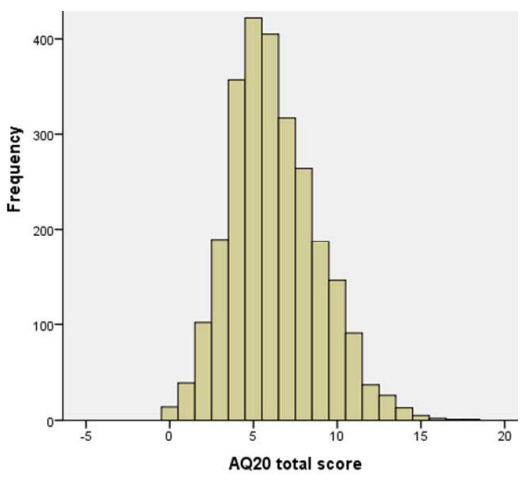


If the study had relied on staff or self-referral alone, rates among the entire prison population would have been just $1 \%$ screening positive for ASD and $0.3 \%$ meeting diagnostic criteria. Of those referred to the study, $85 \%$ had arrived at the prison more than four weeks prior to their referral indicating a delay in identification when this method is used. Referred participants had a significantly higher AQ-20 score $(9.06, \mathrm{SD}=3.11)$ compared with those approached on the prison wings (mean difference $=-2.99,95 \% \mathrm{CI}$ : -3.81 to $-2.18, \mathrm{p}<0.001$ ). Perhaps indicating that prisoners with a more subtle presentation of ASD are more likely to go unrecognised unless they are screened using a standardised assessment. Then again, referred participants were more likely to screen positive for ASD (52\%) and meet diagnostic criteria $(16 \%)$ than those who were approached on the prison wings. Thus, this method may be more efficient at recognising prisoners with ASD; eight who met diagnostic criteria would have been identified using this method alone and four would have been missed.

Unrecognised ASD

Of the 11 prisoners who met the study diagnostic criteria for ASD, six had received specialist input at school, three had been recognised as having ASD in the community but just two were recognised as having ASD by the prison (i.e. an ASD diagnosis was noted in their prison or healthcare record). Three participants had not had any recognition of ASD as far as the researchers could tell from their self-report and a review of their prison healthcare records.

\section{Discussion}

There is great concern about the under-recognition of ASD and evidence that increasing identification would lead to improved outcomes and lower costs to the economy (National Audit Office 2009). Our study found a considerable number of male prisoners who had significant levels of ASD traits some of whom had not been recognised by community or prison services. ASD traits appeared to be normally distributed among our sample and the mean total score was not significantly different than that found in a national sample of male adults of the same age range. Furthermore, the proportion of prisoners who were approached on the prison wings (as opposed to being referred to the study) with ASD was consistent with the estimated prevalence of ASD among males in the community (2\%) (Brugha et al. 2009a).

As such, when diagnostic criteria are applied, it does not appear that there are higher rates of ASD among prisoners compared with the general population. However, based on this rate of $2 \%$, we calculate that there are around 1600 men and 120 women with ASD currently in prisons in the UK (Ministry of Justice, 2015). Up to 25000 UK prisoners are likely to be affected by some level of neuro-disability including ASD, intellectual disability, learning difficulties, learning disorders and ADHD (Underwood et al., 2013). We know these individuals are socially disadvantaged and vulnerable in the community and while in prison (Loucks 2007, Talbot 2008). They may also be at risk of developing mental health problems and self-harm, particularly if their needs are unrecognised and unmet.

The screening tools used by this study was based on the original 50-item AQ which was designed to screen for high-functioning ASD and Asperger syndrome in adults (Baron-Cohen et al. 2006). A shortened version - the AQ20 was developed for a study on the prevalence of 
ASD in the UK (Brugha et al. 2009). At that time, Brugha and colleagues (2009b) considered the AQ to be the most reliable and valid self-report tool available. When the AQ and AQ20 are self-rated they are only suitable for adults with high-functioning ASD where as our study has shown that those with lower levels of functioning and literacy are able to complete the AQ items if they are used in a face-to-face interview. Having said that, in our study, the AQ20 resulted in a high number of false positives and missed three prisoners who self-reported a diagnosis of ASD. More data are needed on whether certain items within the AQ-20 are better predictors of ASD than others or whether a different cut-off is needed in forensic settings.

The ADOS is the only standardised diagnostic assessment that requires direct contact and observation of the individual being assessed for ASD. Most ASD assessment tools used for adults rely on informant-derived data (Sappok et al., 2015). The ADOS is also the only measure able to indicate presence or absence of ASD without the need for any information on a person's developmental history. Given this it would appear to be the most suitable diagnostic assessment for use with prisoners. In our study, the ADOS had to be adapted in order to use it in the prison. Given that this was an ad-hoc adaption we cannot be confident that it has the same reliability and validity as the full ADOS assessment. It would be useful to explore its validity against clinical diagnosis or another 'gold standard' assessment in a prison setting and in a community sample population. Some validation against the ADI-R was attempted but this was not successful due to considerable difficulties in contacting informants who could provide a developmental history (usually a parent). It was interesting that this was not due to unwillingness among participants to provide us with contact details for a parent or consent to us contacting them. Rather, we experienced a lack of engagement from the parents and were only able to carry out three ADI-R assessments. As such, this does not seem to be a feasible way of carrying out diagnostic ASD assessment among prisoners.

\section{Limitations and strengths}

The study took place in one prison in London, UK; limiting the generalisability of the results beyond this setting. A further limitation is that the researchers carrying out the diagnostic assessments were the same people who carried out the screening interviews. As such, they were not blind to participants' screening results and they may have built up a rapport with participants that went on to affect the ADOS assessment. Much of the data collected during the study was reported to the researchers by the participants themselves. Its accuracy may have been affected by recall bias or malingering and by differences in participants' behaviour while in prison compared with when they are in the community.

The inclusion of prisoners who were referred to the study by staff or by themselves introduced recruitment bias to the overall sample of 240 participants. These participants were highly likely to exceed the cut-off on the AQ-20 because they had been recognised as having some ASD traits. This was taken into account by removing these 54 participants from some of the analyses and focussing instead on the 186 participants who were approached on the prison wings. A further limitation of the study is that those approached on the wings were not randomly selected for the study. This was not feasible given the limited resources of the study 
and access to the prisoners. Finding specific prisoners on the prison wings (for follow-up) proved difficult given the amount of time spent on and priority given to educational/training activities. Prisoners did not welcome being approached during 'association' sessions and tended to be dispersed across the wing during this time rather than in their cells.

The prison in which the study took place has a high proportion of prisoners from Black and minority ethnic (BME) backgrounds (around 50\%; Her Majesty's Chief Inspector of Prisons, 2013). However, the AQ-50 and AQ-20 were validated and standardised on populations with much lower rates of people from these backgrounds (Brugha et al., 2009b). The performance of screening/diagnostic tools is under-researched (NICE, 2014) and the prevalence of ASD among BME populations warrants further investigation (Zaroff \& Uhm, 2011). There are a lack of screening tools for adults that have been validated on representative populations (Sappok et al., 2015). However, the AQ has been found to be suitable for measuring ASD traits among specialist populations (Ruzich et al., 2015).

The main strength of the study was the use of standardised assessment tools which were administered in face-to-face interviews by researchers with expertise in ASD. Other studies have sought to obtain information on ASD using prison staff as informants (Robinson et al 2012) or self-rating screening tools (Fazio et al., 2012). These studies have tended to find lower rates of ASD traits than the current study. However, we acknowledge that this level of resource is unlikely to be available to most services in the CJS. A further strength was the level of engagement and rapport that the research team was able to establish within the prison. Healthcare staff welcomed the opportunity to refer prisoners for ASD assessment; something that had not been previously available given the knowledge and experience of current staff.

Core to the project's success was its integration into existing referral and assessment pathways within the prison including access to prisoners' electronic health records so that healthcare staff could refer to the project and the research team could make onward referrals to the full range of available healthcare services in the prison. Having said that, there were problems with the referral pathway for prisoners who met the criteria for ASD. Healthcare staff were keen to refer into the project but found it difficult to know what to do when a prisoner was referred back to them with a diagnosis. Initially, the in-reach team were not willing to accept referrals of prisoners who did not have serious mental illness and there was concern over whether they would be able to provide clinical follow-up for ASD. Healthcare staff were not aware of local health and social care services that might be available to prisoners with ASD. There was no one in the prison who could carry out further assessment and whilst a community referral pathway does exist there is limited access to specialist assessment and intervention once out of prison and no onward referral pathway that could be used to obtain this while a prisoner was in the prison or when they were released into the community.

At a local level, the project successfully demonstrated to the prison that there was a gap in current health screening protocols, diagnostic assessment and intervention for people with ASD. The project has an ongoing legacy in that, following feedback of the results to local 
forensic mental health services, a psychiatrist from the in-reach team was assigned to carry out ASD assessment. Furthermore, an initial assessment form given to prisoners by the prison education centre now gives prisoners an opportunity to report they have ASD (previously they were asked specifically about learning difficulties but not ASD). During the course of the study, there were eight prisoners with ASD that we identified, assessed, referred on to prison mental health services and provided information to GPs, who had previously gone unrecognised in prison and in the community.

Recommendations from this study fall into three categories: staff training, clinical practice and service development. CJS staff should receive training to improve their awareness and understanding of ASD (Alley, 2015). This would be in keeping with national policy on adults with autism titled: Think Autism: An Update (Department of Health, 2014) which has one of the priorities to address the challenges faced by adults with ASD across the CJS with an specific action on the training of prison staff. However there are still considerable gaps in the delivery of national policy and guidance for adults with autism (McCarthy et al., 2015a,b) In addition healthcare staff working within the CJS including prisons should receive enhanced training on assessment and interventions for offenders with ASD as highlighted in a recent review (Robertson \& McGillivray, 2015). Offenders suspected of having ASD should as a result of this enhanced training. CJS healthcare staff should receive enhanced training on assessment and intervention for ASD. Offenders suspected of having ASD should receive further assessment to see whether they meet eligibility for specialist ASD services, screen for other neurodevelopmental disorders including ADHD \& learning disability and to diagnose and treat additional mental health problems. Offenders with ASD should receive specialist input at the earliest stages of the CJS, in order to improve social outcomes including employment and housing, prevent mental health problems and suicidality/self-harm and reduce reoffending.

\section{Conclusion}

Improvements in prison health care should include better recognition of ASD, and the detection (and appropriate treatment) of co-occurring mental health or behavioural problems. This needs to be part of a training programme that is tailored specifically to different prison staff groups. For example the training needs of discipline staff would need to be more specific and cover how autism presents differently within individuals and include examples of how behaviours associated with autism can present in individuals and the best way to support them and develop relationships. The expectation of the Equalities Act 2010 would be that prison staff will be trained to make reasonable adjustments around the communication, sensory and social needs of prisoners with ASD. Initiatives are needed to improve the recognition of ASD further back in the CJS. However, this must be followed up with the provision of interventions that promote liaison and diversion. This should be achieved through the development of a new integrated pathway through the CJS for people with ASD by establishing partnerships between health/social care providers and offender management services. Future reviews and update to policy and practice guidelines on CJS for people with mental illness and learning disability should include ASD. This should include recommendations for stronger links and information sharing protocols between local health, 
social care and local authority for prisoners pre-release to make integrated pathways a reality with the aim of providing appropriate support and reduce the risk of future offending.

\section{References}

Allely, C. S. (2015). Experiences of prison inmates with autism spectrum disorders and the knowledge and understanding of the spectrum amongst prison staff: a review. Journal of Intellectual Disabilities and Offending Behaviour, 6(2), 55-67

Baron-Cohen, S, Wheelwright, S, Skinner, R, Martin, J \& Clubley, E (2001). "The AutismSpectrum Quotient (AQ): Evidence from Asperger Syndrome/High-Functioning Autism, Males and Females, Scientists and Mathematicians." Journal of Autism and Developmental Disorders 31(1): 5-17.

Baron-Cohen, S, Hoekstra, RA, Knickmeyer, R \& Wheelwright, S (2006). "The AutismSpectrum Quotient (AQ)--adolescent version." J Autism Dev Disord 36(3): 343-350.

Bradley, K (2009). The Bradley Report. Lord Bradley's review of people with mental health problems or learning disabilities in the criminal justice system. London, Department of Health.

Brugha, T, McManus, S, Bankart, J, Scott, F, Purdon, S, Smith, J, Bebbington, P, Jenkins, R \& Meltzer, H (2011). "Epidemiology of autism spectrum disorders in adults in the community in England." Archives of General Psychiatry 68(5): 459-466.

Brugha, T, McManus, S, Meltzer, H, Smith, J, Scott, FJ, Purdon, S, Harris, J \& Bankart, J (2009a). Autism Spectrum Disorders in adults living in households throughout England. Report from the Adult Psychiatric Morbidity Survey 2007. London.

Brugha, T, McManus, S, Meltzer, H, Purdon, S, Scott, F, Baron-Cohen, S, Wheelwright, S, Smith, S \& Bankart, J (2009b). Development and testing of methods for identifying cases of Autism Spectrum Disorder among adults in the Adult Psychiatric Morbidity Survey 2007. London.

Chaplin E McCarthy J \& Underwood L, (2013). Autism spectrum conditions and offending: an introduction to the special edition Journal of Intellectual Disabilities and Offending Behaviour, 4 (1/2), $5-8$.

Constantino, JN \& Todd, RD (2003). "Autistic Traits in the General Population a Twin Study." Archives of General Psychiatry 60: 524-530.

Department of Health (2014), Think Autism. Fulfilling and Rewarding Lives, the strategy for Adults with Autism in England: An Update, Department of Health,Hayes, SC (2002). "Early Intervention or Early Incarceration? Using a Screening Test for Intellectual Disability in the Criminal Justice System." Journal of Applied Research in Intellectual Disabilities 15(2): 120-128.

Fazio, R.L, Pietz, C.A, \& Denney, R.L (2012). An estimate of the prevalence of autismspectrum disorders in an incarcerated population. . Open Access Journal of Forensic Psychology, 4, 69-80.

Hayes, S.C. (2002). Early Intervention or Early Incarceration? Using a Screening Test for Intellectual Disability in the Criminal Justice System. Journal of Applied Research in Intellectual Disabilities 15(2), 120-128. 
Her Majesty's Chief Inspector of Prisons. (2013). Report on an unannounced full followup inspection of HMP Brixton 1-12 July 2013 by HM Chief Inspector of Prisons. London.

Hare DJ, Gould J., Mills R. and Wing L. (1999) A Preliminary Study of Individuals with Autistic Spectrum Disorders in Three Special Hospitals in England. London : National Autistic Society.

Haw, Camilla; Radley, Jane; Cooke, Louise. Characteristics of male autistic spectrum patients in low security: are they different from non-autistic low secure patients? Journal of Intellectual Disabilities and Offending Behaviour, Volume 4, Numbers 1-2, 2013, pp. 2432(9)

Hoekstra, RA, Vinkhuyzen, AAE, Wheelwright, S, Bartels, M, Boomsma, DI, BaronCohen, S, Posthuma, D \& Sluis, S (2010). "The Construction and Validation of an Abridged Version of the Autism-Spectrum Quotient (AQ-Short)." Journal of Autism and Developmental Disorders 41(5): 589-596.

Holden, B \& Gitlesen, J (2006). "A total population study of challenging behaviour in the county of Hedmark, Norway: Prevalence, and risk markers." Research in Developmental Disabilities 27(4): 456-465.

King C, Murphy GH. A systematic review of people with autism spectrum disorder and the criminal justice system. J Autism Dev Disord. 2014 Nov;44(11):2717-33. doi: 10.1007/s10803-014-2046-5.

Ledingham R, Mills, R. (2015) "A preliminary study of autism and cybercrime in the context of international law enforcement", Advances in Autism, Vol. 1 Iss: 1, pp.2-11

Lewis A, Pritchett r, Hughes C, Turner K. (2015). Development and implementation of autism standards for prisons. Journal of Intellectual Disabilities and Offending Behaviour. 6(2), 68-80.

Levy, SE, Mandell, DS \& Schultz, RT (2009). "Autism." Lancet 374: 1627-1638.

Lord, C., Rutter, M., Goode, S., Heemsbergen, J., Jordan, H., Mawhood, L., \& Schopler, E. (1989). Autism diagnostic observation schedule: a standardized observation of communicative and social behavior. J Autism Dev Disord, 19(2), 185-212.

Lord, C., Rutter, M., \& Le Couteur, A. (1994). Autism Diagnostic Interview-Revised: revised version of a diagnostic interview for caregivers of individuals with possible pervasive developmental disorders. Journal of Autism and Developmental Disorders, 24(5), 659-685.

Loucks, N (2007). No One Knows: offenders with learning difficulties and learning disabilities - review of prevalence and associated needs. London.

Ministry of Justice (2009). NOMS London Strategic Commissioning Plan 2010-2013. London.

Ministry of Justice (2015). Prison population figures, population bulletin: weekly 7 August 2015. Accessed online on 13th August 2015 at: https://www.gov.uk/government/statistics/prison-population-figures-2015

McCarthy, J., Underwood, L., Chaplin, E., Hayward, H., Sabet, J., ...Murphy, D. (2015a). Screening and diagnostic assessment of neurodevelopmental disorders in a male prison. Journal of Intellectual Disability and Offending Behaviour. 6 (2)

McCarthy, J., Underwood, L., Chaplin, E., Hayward, H., Sabet, J., ...Murphy, D. (2015b). Characteristics of prisoners with neurodevelopmental disorders. Journal of Intellectual Disability Research 
McCarthy J, Chaplin E, Underwood L. (2015c). An English perspective on policy for adults with autism. Advances in Autism, 1(2), 61-65.

National Audit Office (2009). Supporting people with autism through adulthood. Model to assess the financial impacts of providing multi-disciplinary support services for adults with high-functioning autism/Asperger syndrome. Technical Paper. London.

National Audit Office (2009). Supporting people with autism through adulthood. Report by the Comptroller and Auditor General. HC 556 Session 2008-2009. London.

National Centre for Social Research and University of Leicester, Adult Psychiatric Morbidity Survey, 2007 [computer file]. 3rd Edition. Colchester, Essex: UK Data Archive [distributor], January 2011. SN: 6379, http://dx.doi.org/10.5255/UKDA-SN-6379-1

NICE. (2014). Autistic spectrum disorders in adults. London: National Institute for Health and Clinical Excellence

Offender Health Collaborative (2012). National Liaison and Diversion Development Network contract. London, The Home Office.

Robinson L, Spencer MD, Lindsay DG, Stanfield AC, Owens DGC, Hall J \& Johnstone EC. (2012). Evaluation of a Screening Instrument for Autism Spectrum Disorders in Prisoners. PLoS ONE 7 (5): e36078. Doi: 10.1371/journal.pone0036078

Robertson, C. E., \& McGillivray, J. A. (2015). Autism behind bars: a review of the research literature and discussion of key issues. The Journal of Forensic Psychiatry \& Psychology, 26(6), 719-736.

Sabet, J., Underwood, L., Chaplin, E., Hayward, H., McCarthy, J. (In press). Autism Spectrum Disorder, Attention-Deficit Hyperactivity Disorder and Offending. Advances in Autism 1 (2)

Sappok, T., Heinrich, M., Underwood, L. (2015). Screening tools for autism spectrum disorders. Advances in Autism, 1 (1), 12-29.

Siponmaa L, Kristiansson M, Jonson C, Nydén A, Gillberg C. (2001). Juvenile and young adult mentally disordered offenders: the role of child neuropsychiatric disorders. J Am Acad Psychiatry Law 29(4):420-6.

Søndenaa, E, Rasmussen, K, Palmstierna, T \& Nøttestad, J (2008). "The prevalence and nature of intellectual disability in Norwegian prisons." Journal of Intellectual Disability Research 52(12): 1129-1137.

Talbot, J (2008). No One knows report and final recommendations: Prisoners voices Experiences of the criminal justice system by prisoners with learning disabilities and difficulties. London, Prison Reform Trust.

Tsiouris, JA, Cohen, IL, Patti, PJ \& Korosh, WM (2003). "Treatment of previously undiagnosed psychiatric disorders in persons with developmental disabilitis decreased or eliminated self-injurious behavior." Journal of Clinical Psychiatry 64: 1081-1090.

Underwood, L., Forrester, A., Chaplin, E., \& McCarthy, J. (2013). Prisoners with neurodevelopmental disorders. Journal of Intellectual Disabilities \& Offending Behaviour, 4, $17-23$.

Zaroff, C., \& Uhm, S.Y. (2011). Prevalence of Autism Spectrum Disorders and Influence of Country of Measurement and Ethnicity. Social Psychiatry 47(3):395-8. 